HARDY-RAMANUJAN JOURNAL 34 \& 35 (2013), 11-17

\title{
THE WORK OF K. RAMACHANDRA IN ALGEBRAIC NUMBER THEORY
}

\author{
M. RAM MURTY
}

\section{INTRODUCTION}

The division of knowledge into disciplines and sub-disciplines is a human construction. Nature knows no such divisions. It is the limitation of the human mind that forces us to make such divisions so that we are not overwhelmed by the enormity of the subject. But this we forget and we become prisoners of our own thoughts and concepts.

Truly, there is no division between algebraic number theory and analytic number theory. They are convenient (and temporary) labels that refer to certain aspects and methods of the larger domain of number theory, which too cannot be so easily separated from other disciplines of scientific knowledge. The relationship between analytic and algebraic number theory is symbiotic as in a complete selfsustaining organism. Ramachandra's early work classified as algebraic number theory really contains elements of analytic number theory. There are elements of algebraic number theory in his work on transcendental numbers as well, but we don't discuss that here. Having said this, we will discuss primarily his three papers in algebraic number theory since this is what the editors of the Hardy-Ramanujan Journal (which Ramachandra founded) have asked me to do.

Kanakanahalli Ramachandra did not write many papers in algebraic number theory. But the few that he wrote in this area are gems and comprise now fundamental works in the field. His doctoral thesis, modestly titled "Some applications of Kronecker's limit formula" was published in the Annals of Mathematics. It contains remarkable and new results on the construction of ray class fields of an imaginary quadratic field. The paper also constructs a set of units, now called Ramachandra units (or elliptic units) that generate these class fields in a functorial way. In addition, the paper has striking results regarding special values of modular forms with algebraic Fourier coefficients, evaluated at CM points. Independently, Ramachandra had obtained the celebrated Chowla-Selberg formula (announced by Chowla and Selberg in 1949 [1] and published later by them with 
detailed proofs in 1967 [2]). Ramachandra's construction of these ray class fields using his units has since been simplified by Schertz [10].

His second paper [8] dealt with units of cyclotomic fields. Upon reading this paper carefully, it becomes clear that it was partly inspired by his first paper [7]. His third paper [9] can be viewed as an extension of both of these papers and concerns relative abelian number fields.

\section{KRONECKER'S LIMIT FORMULA}

Ramchandra's work on applications of Kronecker's limit formula was based on Siegel's lectures [11] delivered at the Tata Institute of Fundamental Research in Mumbai, India during the winter semester of 1959/60. The aim of these lectures was to introduce to students "some of the important and beautiful ideas which were developed by L. Kronecker and E. Hecke," in Siegel's own words. The notes were written up by S. Raghavan.

Let $\Delta(z)$ be Ramanujan's cusp form:

$$
\Delta(z)=q \prod_{n=1}^{\infty}\left(1-q^{n}\right)^{24}, \quad q=e^{2 \pi i z} .
$$

Let $K$ be an imaginary quadratic field and let $\mathfrak{b}$ be an ideal of $\mathcal{O}_{K}$. If $\left[\beta_{1}, \beta_{2}\right]$ is an integral basis of $\mathfrak{b}$, with $\Im\left(\beta_{2} / \beta_{1}\right)>0$, we define

$$
g(\mathfrak{b}):=(2 \pi)^{-12}(\mathbf{N}(\mathfrak{b}))^{6} \Delta\left(\beta_{1}, \beta_{2}\right),
$$

where

$$
\Delta\left(\omega_{1}, \omega_{2}\right)=\omega_{1}^{-12} \Delta\left(\omega_{2} / \omega_{1}\right)
$$

As shown by Ramachandra (see p. 109 of [7]), $g(\mathfrak{b})$ is well-defined and does not depend on the choice of an integral basis of $\mathfrak{b}$. In fact, it depends only on the ideal class that $\mathfrak{b}$ belongs to in the ideal class group of $K$. Thus, if $\mathcal{C}$ denotes an ideal class and $\mathfrak{b} \in \mathcal{C}$, we denote $g(\mathfrak{b})$ by $g(\mathcal{C})$, there being no confusion. This is the content of Lemma 2 of [7].

Let $d_{K}$ be the discriminant of $K$ and let $w$ be the number of roots of unity in $\mathcal{O}_{K}$. We write

$$
\zeta(s, \mathcal{C}):=\sum_{\mathfrak{a} \in \mathcal{C}} \mathbf{N}(\mathfrak{a})^{-s}
$$

for the ideal class zeta function. Hecke showed that $\zeta(s, \mathcal{C})$ extends analytically to the whole complex plane except for $s=1$ where it has a simple pole. Kronecker's 
first limit formula gives the residue and the constant term of the Laurent expansion of $\zeta(s, \mathcal{C})$ at $s=1$ :

$$
\zeta(s, \mathcal{C})=\frac{2 \pi}{w \sqrt{\left|d_{K}\right|}}\left(\frac{1}{s-1}+2 \gamma-\log \left|d_{K}\right|-\frac{1}{6} \log \left|\mathfrak{g}\left(\mathcal{C}^{-1}\right)\right|\right)+O(s-1),
$$

as $s \rightarrow 1+$. Ramachandra uses this analytic formula quite deftly to derive a gallery of beautiful results that properly belong in the domain of algebraic number theory. To describe these, we introduce the classical $\vartheta$-function. Let

$$
\vartheta(z ; u, v)=\sum_{n=-\infty}^{\infty} e^{\pi i z(n-v)^{2}+2 \pi i n u+\pi i u v}
$$

with rational $u, v$ and common denominator $f$. Put

$$
\Phi(z ; u, v)=\left\{\vartheta(z ; u, v) \eta(z)^{-1}\right\}^{12}
$$

where $\eta(z)$ is the classical $\eta$-function of Dedekind. A basic result of Kronecker is the modular transformation rule:

$$
\Phi^{f}(M z ; M(u, v))=\Phi^{f}(z ; u, v)
$$

for all $M \in S L_{2}(\mathbb{Z})$.

If $K$ is an imaginary quadratic extension of the rational number field $\mathbb{Q}$, we will also denote by $d$ its discriminant $d_{K}$ and $\mathfrak{d}$ its different. If $\mathfrak{f}$ is an ideal, we denote by $\overline{\mathfrak{f}}$ its conjugate ideal. We let $K_{\mathfrak{f}}$ and $K_{\overline{\mathfrak{f}}}$ be the class fields of $K$ with ray class groups $(\bmod \mathfrak{f})$ and $(\bmod \overline{\mathfrak{f}})$ respectively. For a divisor $\mathfrak{g}$ of $\mathfrak{f}$, and ray class $R(\bmod$ $\mathfrak{f})$, put

$$
\Phi_{\mathfrak{f}, \mathfrak{g}}(R)=\Phi^{f}\left(\beta_{2} / \beta_{1} ; \operatorname{Tr}\left(\beta_{1}\right), \operatorname{Tr}\left(\beta_{2}\right)\right),
$$

where $\beta_{1}, \beta_{2}$ is a basis of $\mathfrak{b} \mathfrak{g} / \mathfrak{d} \mathfrak{f}$ with $\mathfrak{b}$ being an ideal of the ray class $R$. This is an invariant of $R$. For a character $\chi$ of the ray class group, we denote by $\mathfrak{f}_{\chi}$ its conductor. Kronecker's limit formula now yields for $\chi \neq 1$,

$$
\sum_{R} \bar{\chi}(R) \log \left|\Phi_{\mathfrak{f}, \mathfrak{g}}(R)\right|=0
$$

if $\mathfrak{g}$ does not divide $\mathfrak{f} / \mathfrak{f}_{\chi}$ and $r(\mathfrak{f}, \mathfrak{g}, \chi) L\left(1, \chi^{*}\right)$ otherwise where $r(\mathfrak{f}, \mathfrak{g}, \chi)$ is non-zero elementary factor and the sum is over all ray classes $R(\bmod \mathfrak{f})$. For the trivial character, we have

$$
\sum_{R} \log \left|\Phi_{\mathfrak{f}, \mathfrak{g}}(R)\right|=0
$$

if $\mathfrak{f} / \mathfrak{g}$ is not a power of a prime ideal and is $r(\mathfrak{f}, \mathfrak{g}) L_{d}(1)$ otherwise where

$$
L_{d}(s)=\sum_{n=1}^{\infty}(d / n) n^{-s}
$$


is the classical Dirichlet $L$-series attached to the quadratic character $\chi_{d}:=(d / \cdot)$ and $r(\mathfrak{f}, \mathfrak{g})$ is a non-zero elementary factor.

The remarkable theorem proved by Ramachandra is that the numbers $\Phi_{\mathfrak{f}, \mathfrak{g}}(R)$ are conjugate algebraic numbers contained in the ray class field $K_{\bar{f}}$. Moreover, these are all units if $\mathfrak{g}=(1)$ and $\mathfrak{f}$ is a power of prime ideal. Otherwise, suitable power products $\epsilon(R)$ of them are units. The expert will note the similarity to the situation in the cyclotomic fields (discussed in the next section). In addition, one can describe the action of the Galois group explicitly: for $\sigma \in G=\operatorname{Gal}\left(K_{\overline{\mathfrak{f}}}\right)$,

$$
\Phi_{\mathfrak{f},(1)}(R)^{\sigma}=\Phi_{\mathfrak{f},(1)}\left(R \bar{S}_{\sigma}\right) \quad \epsilon(R)^{\sigma}=\epsilon\left(R \bar{S}_{\sigma}\right),
$$

where $S_{\sigma}$ is the ray class mod $\overline{\mathfrak{f}}$ associated to $\sigma$ via class field theory. Using Hasse's theory of complex multiplication [5], Ramachandra shows that for any subfield field $K$ of $K_{\overline{\mathfrak{f}}}$, the relative norm $N_{K_{\bar{f}} / K}(\epsilon(R))$ generates $K$.

These units have other remarkable properties. For instance, the subgroup generated by the units $\epsilon(R)^{\sigma-1}$ as $\sigma$ ranges over elements of the Galois group $G$, has finite index in the group of total units of $K_{\bar{f}}$ and this index can be given explicitly in terms of the class number of $K_{\bar{f}}$, the class number of $K$, the number of ray classes mod $\mathfrak{f}$ and the generalized Euler function. This formula generalizes earlier work of Fueter [3] and Siegel [11].

The final section of the paper focuses on the field generated by special values of modular forms evaluated at CM points. These values are often called singular values in the literature. In this section, Ramachandra rediscovers the celebrated Chowla-Selberg formula:

$$
\prod_{\mathcal{C} \in \mathcal{H}_{K}} g(\mathcal{C})^{1 / 3}=\left(\frac{1}{2 \pi\left|d_{K}\right|}\right)^{2 h} \prod_{a=1}^{d} \Gamma(a / d)^{w \chi_{d}(a)} .
$$

In fact, it is possible to express the value of Ramanujan's cusp form $\Delta(z)$ and its derivatives at any point $z$ in the upper half-plane such that $\mathbb{Q}(z)$ is an imaginary quadratic field. For instance, Ramachandra gives the pretty formula

$$
\Delta\left(\frac{d+\sqrt{2}}{2}\right)=(-1)^{d}\left(\frac{2 \pi}{|d|}\right)^{6} \prod_{a=1}^{|d|} \Gamma(a /|d|)^{3 \chi_{d}(a)} .
$$

Thus, putting $d=-3,-4,-7,-8$ respectively, he finds

$$
\begin{gathered}
\Delta\left(\frac{1+\sqrt{-3}}{2}\right)=\left(\frac{2 \pi}{3}\right)^{6} \Gamma(1 / 3)^{18} \Gamma(2 / 3)^{-18}, \\
\Delta(\sqrt{-1})=\left(\frac{\pi}{2}\right)^{6} \Gamma(1 / 4)^{12} \Gamma(3 / 4)^{-12},
\end{gathered}
$$




$$
\Delta\left(\frac{1+\sqrt{-7}}{2}\right)=\left(\frac{2 \pi}{7}\right)^{6} \Gamma(1 / 7)^{6} \Gamma(2 / 7)^{6} \Gamma(4 / 7)^{6} \Gamma(3 / 7)^{-6} \Gamma(5 / 7)^{-6} \Gamma(6 / 7)^{-6},
$$

and

$$
\Delta(\sqrt{-2})=\left(\frac{\pi}{4}\right)^{6} \Gamma(1 / 8)^{6} \Gamma(3 / 8)^{6} \Gamma(5 / 8)^{-6} \Gamma(7 / 8)^{-6}
$$

Ramachandra notes the elegance of these formulas is due to the fact that the corresponding class number is 1 . If the class number is greater than 1 , the formula takes the following shape:

$$
\prod_{a, b, c} a^{-6} \Delta\left(\frac{b+\sqrt{d}}{2 a}\right)=(2 \pi|d|)^{-6 h} \prod_{m=1}^{|d|} \Gamma(m /|d|)^{3 \chi_{d}(m) w}
$$

where the product on the left hand side is over a complete set of reduced binary quadratic forms of fundamental discriminant $d<0$.

Recently, the work of Ramachandra had been used by the author and V. Kumar Murty [6] to obtain new transcendence results regarding special values of $L$-series attached to the ideal class groups. Related to the results on singular values, Gun, Murty and Rath [4] have obtained transcendence results regarding these special values. Clearly, more knowledge can be gleaned from this fundamental paper. As already noted, Ramachandra's paper is an impressive work and is the most cited of all of his papers on MathSciNet.

\section{UNITS OF CYCLOTOMIC FIELDS}

Let $\zeta$ be a primitive $q$-th root of unity. The unit group of $\mathbb{Q}(\zeta)$ has $\operatorname{rank} \frac{1}{2} \varphi(q)-1$. When $q$ is a prime or a prime power, it is easy to construct a maximal system of multiplicatively independent units. However, when $q$ is not a prime power, it was an open problem (until Ramachandra's paper) to write down an explicit set of such units. Ramachandra's construction is easy and elegantly described as follows. Put

$$
u_{s}=\frac{1-\zeta^{s}}{1-\zeta}, \quad 1<s<q / 2, \quad(q, s)=1 .
$$

If $q$ is a prime power, these numbers comprise a maximal set of independent units. However, if $q$ is not a prime power, Ramachandra showed that there are values of $q$ for which these numbers are multiplicatively dependent (thus answering negatively a question posed by Milnor in a letter dated February 6, 1964 and written to K.G. Ramanathan who was the official doctoral supervisor of Ramachandra). However, Ramachandra shows one can make the following construction. Let

$$
q=\prod_{i \in I} p_{i}^{a_{i}}
$$


be the unique factorization of $q$ into prime powers and the $p_{i}$ 's distinct. For each subset $J$ of $I$, put

$$
q_{J}=\prod_{j \in J} p_{j}^{a_{j}}, \quad \text { and } \quad \zeta_{J}=\zeta^{q_{J}} .
$$

Then, Ramachandra [8] proved that

$$
v_{s}:=\prod_{J \subseteq I} \frac{1-\zeta_{J}^{s}}{1-\zeta_{J}}, \quad 1<s<q / 2, \quad(q, s)=1
$$

comprise a maximally independent set of units. For the cognoscenti, one can see a structural similarity between this paper and Ramachandra's Annals paper. In both papers, a fundamental system of units is constructed for certain types of ray class fields, namely those of either an imaginary quadratic field or the rational number field.

\section{Class numbers of RElative ABElian FiElds}

Ramachandra wrote [9] yet one more paper in which he derives a Kummer type formula for the class number of abelian extensions of any totally imaginary number field. To do this, he uses work of Hecke who generalized Kronecker's first limit formula for arbitrary algebraic number fields. These results again can be used to give a maximal system of independent units of any absolute abelian number field. The results here are a bit complicated to state. However, it is clear they have structural similarity to his two earlier papers and are inspired by the latter.

Without a doubt, these works have inspired further developments, the most notable being Stark's conjectures on special values of Artin $L$-series [12].

\section{REFERENCES}

[1] S. Chowla and A. Selberg, On Epstein's zeta function, I, Proc. Nat. Acd. Sci. U.S.A., 35 (1949), 371-374.

[2] S. Chowla and A. Selberg, On Epstein's zeta function, J. Reine Angew. Math., 227 (1967), 86-110.

[3] R. Feuter, Die verallgemeinerte Kroneckersche Grenzformel und ihre Anwendung auf die Berechnung der Klassenzahl, Rend. Circ. Mat. Palermo, 29 (1910), 380-395.

[4] S. Gun, M. Ram Murty and P. Rath, Algebraic independence of values of modular forms, International Journal of Number Theory, 7 (2011), 1065-1074.

[5] H. Hasse, Neue Begründung der komplexen Multiplikation, Teil I, J. Reine Angew. Math., 157 (1926), 115-139 and Teil II, 165 (1931), 64-88.

[6] M. Ram Murty and V. Kumar Murty, Transcendental values of class group $L$-functions, Math. Annalen, 351 (2011), 835-855. 
[7] K. Ramachandra, Some applications of Kronecker's limit formulas, Annals of Math., 80 (2) (1964), 104-148.

[8] K. Ramachandra, On the units of cyclotomic fields, Acta Arithmetica, 12 (1966/67), 165-173.

[9] K. Ramachandra, The class number of relative abelian fields, J. Reine Angew. Math., 236 (1969), 1-10.

[10] R. Schertz, Construction of ray class fields by elliptic units, J. Théor. Nombres Bordeaux, 9 (1997), no. 2, 383-394.

[11] C.L. Siegel, Advanced Analytic Number Theory, Tata Institute Lecture Notes, 1962.

[12] H.M. Stark, Values of $L$-functions at $s=1$, L-functions for quadratic forms, Advances in Math., 7 (1971), 301-343.

Department of Mathematics, Queen's University, Kingston, Ontario, K7L 3N6, Canada

E-mail address: murty@mast.queensu.ca 